\title{
Technologies for Ocean Worlds
}

\author{
Corresponding Author: Patricia M. Beauchamp \\ pbeaucha@jpl.nasa.gov, 818-645-2479 \\ Jet Propulsion Laboratory, California Institute of Technology \\ For the Outer Planets Assessment Group (OPAG) \\ 2018 Roadmap for Ocean Worlds (ROW) Team
}

\begin{abstract}
Jeff Moore, OPAG Co-chair
Linda Spilker, OPAG Co-chair

Amanda Hendrix, ROW Co-lead

Terry Hurford, ROW Co-lead
\end{abstract}

\section{Co-authors/endorsers:}

$\begin{array}{ll}\text { Alfred McEwen } & \text { Jeff Bowman } \\ \text { Morgan Cable } & \text { Tom Spilker } \\ \text { Abigail Rymer } & \text { Scott Edgington } \\ \text { Britney Schmidt } & \text { Kunio Sayanagi }\end{array}$

\begin{abstract}
This white paper summarizes and provides an overview of the cross-cutting technologies necessary for Ocean Worlds, their status and needed developments. It complements the two white papers arising from the OPAG Roadmap for Ocean Worlds (ROW) committee and updates and condenses the Technology Chapter III of the ROW report, Ocean Worlds: Priorities, Missions Scenarios, \& Technologies [Hendrix, Hurford et al 2018 https://www.lpi.usra.edu/opag/ROW/PrioritiesMissions-Scenarios-0218.pdf], where the details can be found. This white paper does not reiterate the entire ROW report but only provides an overview, with key technologies in italics, followed by an updated status of selected technologies.
\end{abstract}

For Ocean Worlds missions, NASA should continue investing in Europa Lander mission concept technologies and fund key developments both for a Neptune (Triton) flagship mission and for innovative new concepts to land and sample on and below the surface of Ocean Worlds.

\begin{tabular}{|c|}
\hline Priority Technologies for Ocean Worlds \\
\hline High-efficiency Power Subsystems \\
\hline System, Subsystem and Science Autonomy \\
\hline Surface and Sub-surface robotic systems \\
\hline Low-mass, low-power instruments, mechanism and electronics \\
\hline Efficient communications \\
\hline
\end{tabular}

Some of this work was carried out at the Jet Propulsion Laboratory, California Institute of Technology, under a contract with the National Aeronautics and Space Administration (80NM0018D0004). Pre-decisional information for planning and discussion purposes only. 


\section{Introduction}

This white paper discusses the broader capabilities needed to investigate all the Ocean Worlds (OWs), including Triton, as part of a Neptune mission, where landing is not as likely in the next decade. There are also many excellent $O W$ white papers submitted on technologies for surface and sub-surface missions e.g., [Schmidt and Craft 2020], [Carpenter 2020], [Carpenter 2020] and [Crum 2020]. All of these capture the necessary technology developments for specific mission types, although the latter illustrates how Europa Lander is developing technologies that support a variety of OWs missions. There are also many applicable detailed white papers on technologies needed for OWs e.g. Autonomy [Reeves 2020], Additive Manufacturing (AM) [McEnerney 2020], Aerocapture [Austin 2020], and Planetary Protection [Benardini 2020].

\section{Overview}

Key to accomplishing the goals of exploring OWs are technology advances that satisfy the environmental constraints of extremely low temperatures and often high radiation while meeting the stringent planetary protection and contamination control requirements for $\mathrm{OW}$ missions. Reducing the system mass and providing power are critical in all cases. Power sources and energy storage systems suitable for cryogenic environments and autonomous systems are required for all missions envisaged. Since these missions will be far from Earth, autonomous systems and subsystems are key to enabling the aerial or landed functionalities and scientific measurements. To facilitate autonomy, there also needs to be improvements in radiation-hard flight computers and memory. All OW missions have a requirement for cold-compatible, low-mass, low-power instruments, mechanism and electronics with the possible exception of those housed in radiationhard containers, which double as warm enclosures, and even then, some of the instrumentation is external to the vault. For landers and plumes, the sample acquisition and handling mechanisms are at ambient, as are many of the instrument components. Landed missions often demand codevelopment of synergistic sub-systems e.g. in situ organic/life detection and sampling methods with subsurface access technologies and planetary protection/contamination control as well as innovative power and communication subsystems. Mass savings without compromising performance can be achieved through coupling system engineering and additive manufacturing of complex and multi-purpose structures. For flying through plumes, we also need to successfully demonstrate plume capture while sample return missions demand cryogenic containment. Lastly, without communications to the DSN there is no mission, so the need to have reduced mass, power and volume communication elements that efficiently transmit high data rates from/to landed and sub-surface elements (large deployable antennas, low power, GaN solid state power amplifiers) is critical as described in detail in the ROW report. As deeper sub-surface samples are acquired in situ communications technologies are also necessary.

There are many technologies common to all OW missions that can reduce the mission cost and/or risk. Solar Electric Propulsion (SEP) enables missions requiring large in-space velocity changes over time and can often provide enhanced and enabling trajectories to the outer planets or to Ceres. This technology opens up mass and trip-time trades, offering major performance gains and significant improvements to mission capabilities Research in Astrodynamics can open up new types of missions, such as the Voyager Grand Tour or the Cassini Grand Finale and continuing research is vital to innovative mission designs especially for the Ice Giants. Finally, Aerocapture is a technology that has been available for over a decade, but has not been used for a variety of reasons, mainly to avoid perceived risk. Astrodynamics, aerocapture and propulsion sub-systems and launch vehicles can all be traded to find an optimal solution to mission design. 
It cannot be emphasized enough, however, that mass and power are the limiting factors for all OW mission concepts and, in the case of the Jupiter system, radiation; these are what distinguish applicable technologies.

\section{2020 Updates on Mission-Related Technologies}

\subsection{Power Sources}

Outer planet missions, such as Voyager, Cassini, and Galileo, have typically used Radioisotope Power Systems (RPS). But this is beginning to change. For the first time, Juno, a mission to Jupiter, is powered by a solar power system and Europa Clipper will use solar power. Stored energy systems in the form of primary batteries are also viable for short-lived missions and the Europa Lander concept has improved the viability of high specific energy batteries. However, Radioisotope Power Systems (RPSs) are still necessary for the more distant bodies, such as Neptune, in the solar system and/or complicated aerial or landed missions within the Saturnian system and beyond.

\subsubsection{Radioisotope Power Systems (RPS)}

Radioisotope Thermoelectric Generators (RTGs) provide electrical power using heat from the natural radioactive decay of plutonium-238, in the form of plutonium dioxide. At present the Multi-Mission Radioisotope Thermoelectric Generator (MMRTG) is available and has been used on Curiosity and Perseverance missions to Mars. Underlying thermoelectric technologies required to enable a potential enhanced MMRTG (eMMRTG) are also currently in life-test. This enhancement of the MMRTG promises higher available power at end of life and, so far, current life-test results support that conclusion. The eMMRTG could be used for orbital/fly-by missions and could also be used for surface missions on bodies with or without an atmosphere. For Titan, modifications to the MMRTG design are needed to accommodate environmental conditions.

Plans for the next generation of RTGs are being evaluated and this is vital for many OW missions. The current plan calls for a delivery of at least two, possibly three General Purpose Heat Source (GPHS)-RTG using existing hardware for a 2030 launch. A GPHS-RTG uses the same thermoelectrics (SiGe unicouples) as the Multi-Hundred-Watt (MHW) RTG (with a lifetime of 43+ years on Voyagers 1 and 2). For a modest investment, the existing GPHS-RTG qualification unit could be refurbished to provide an unfueled system available for a mission to the Ice Giants, and thereby Neptune and Triton, by the end of 2022. Additionally, available SiGe unicouples could be harvested from units in storage and qualified for risk mitigation and a production line established with an unfueled flight system provided by 2026; this latter scenario is not currently in scope. Maturing new thermoelectric technologies will proceed in tandem with the GPHSRTG refurbishment, albeit at a slower pace than previously planned. This "Mod 2" technology development would be for a true next generation RTG. A GPHS-RTG and the next generation RTG are usable only in vacuum, so for Titan a MMRTG is the only option. The ${ }^{238} \mathrm{Pu}$ production would need to be scaled up for a Neptune launch but that is possible now that the DOE is using a constant-rate production strategy where key equipment has been updated and processes verified.

For landed subsurface/drilling missions, there has been some research and development, e.g. SESAME, funded by PSD to incorporate the next generation RPS technologies into cryobots, which are probes for OW exploration. Without good power systems, access to the sub-surface is impossible. Such RPS developments should be funded to provide the community with deeper subsurface access than Europa Lander is planning. Adoption of a next generation RTG technologies could be enabling for cryobots to travel through ice shells to ice-ocean interfaces [Woerner 2020]. These kinds of systems are long-lead items and deserve NASA's early attention to conduct proof- 
of-concept studies. Funding needs to be started this decade in order for these technologies to be ready before 2040 .

\subsubsection{Solar Power}

Solar-powered NASA missions to Jupiter that are currently flying (Juno)[Stella 2009 and Dawson 2012] or in advanced stages of development (Europa Clipper) [Kroon 2019] make use of state-of-practice lattice-matched triple-junction solar cells. Recently, higher-performance solar cell technologies specifically intended for LILT applications have been developed and demonstrated in ground testing[Chiu 2019 and McPheeters 2018]. One of these, (LILTIMM4)[Chiu 2019], promises to be particularly well suited for Jupiter mission environments, thanks to significantly higher end-of-life efficiency after exposure to radiation, compared to state of practice cells. To facilitate rapid infusion of these new technologies into future Jupiter flight missions, their radiation performance at LILT should be validated through low-temperature, highdose irradiation testing with in-situ characterization.

No solar-powered missions to Saturn have flown yet and none are currently in the advanced stages of development. However, ground testing of commercial off-the-shelf space solar cells including has revealed that they have viable efficiencies even under the extreme LILT conditions of the Saturn system; also, brief excursions to the high temperatures expected during Venus flyby off-nominal events, as may occur on the way to Saturn, were found to not have a measurable effect on these cells' subsequent LILT performance [Boca 2017]. Against this baseline, the recently-developed LILT-optimized cell technologies offer an efficiency boost and, in the case of LILT-IMM4, significantly lower mass, which is particularly relevant given the high specific powers required of Saturn solar arrays [Boca 2019]. Infusing these new solar cell technologies into Saturn flight missions will be accelerated by increasing their TRL, through qualification of their LILT performance in statistically significant sample sets. In spite of these advances, SOP solar power systems are currently not attractive for the future planetary mission concepts to outer planets beyond Saturn, because of limited performance capabilities at low solar irradiance and lowtemperature environments or for high-power solar electric propulsion missions to outer planets, because such solar arrays would be heavy and cumbersome.

\subsubsection{Energy Storage Devices}

The Europa Lander Pre-Project team has a goal to provide a 20- to 30-day mission life for a $100 \mathrm{~kg}$ battery package for the landed system which have to withstand high radiation and meet planetary protection requirements, including a level of sterilization. In addition to power, the batteries dissipate heat that is harvested for thermal management. The study team have initiated early testing focused on $\mathrm{Li} / \mathrm{CF}_{\mathrm{x}}-\mathrm{MnO}_{2}$ chemistries and are including more extensive evaluation of $\mathrm{Li} / \mathrm{CF}_{\mathrm{x}}$ for Lander going forward, focusing more on higher temperatures (0 to $\left.60^{\circ} \mathrm{C}\right)$ [Crum 2020]. Further cell design improvements are underway to improve specific energy. Based on the results so far, they expect to double the surface life from 20 to 40 days, which would also benefit many other OW missions.

\subsection{Astrodynamics, Propulsion and Aerocapture}

Early funding for astrodynamics studies would produce new techniques prior to formulation of missions, which could lead to novel and exciting concepts. Astrodynamics research can also develop ways to apply other spacecraft technology developments to missions, e.g. leveraging the SLS heavy lift capability to enable missions to the far Outer Solar System. Investment in Astrodynamics research would expand NASA's capability for future robotic missions and develop new capabilities for OWs missions, particularly an orbiting Neptune mission. 
Chemical propulsion is a fairly mature technology and its current state enables orbital missions to every giant planet system but improvements in IsP will aid Neptune orbital missions. Development of low-freezing-point bipropellants that can spacecraft power and mass by requiring less heater power to prevent propellants from freezing would also be advantageous. Cold gas propulsion, is potentially useful for landings on airless OWs, or any location with biological or pre-biological potential, due to its ability to avoid chemical contamination of the landing site. Such systems often use nitrogen $\left(\mathrm{N}_{2}\right)$ but other systems using propellants offering higher $\mathrm{I}_{\mathrm{SP}}$ would be advantageous. SEP is used where sunlight is abundant so it can be useful for sending vehicles on transfer trajectories to the outer solar system, but not for maneuvering there.

Astrodynamics dictates that a spacecraft must slow down significantly to enter orbit around a planetary body, or else only perform a single flyby. Historically, orbit insertion has been accomplished using a large propulsion system with a significant amount of propellant onboard the spacecraft, leading to "traditional" spacecraft with more than half of their mass being devoted to propulsion systems and propellants. Aerocapture takes advantage of a naturally occurring resource at many planetary destinations, atmospheric drag, to slow a spacecraft down and enter orbit [Austin 2020, Spilker 2019] and with the recent Thermal Protection Systems (TPS) available, this is viable. Continued funding for the TPS technologies is needed to enable missions such as these.

\subsection{Autonomy}

Autonomous planetary spacecraft systems have long thought to enable more complex missions and/or frequent launches by reducing operational costs. This is especially true of longduration missions on planetary surfaces, such as missions to the OWs. Current spacecraft are sequenced either in time or sometimes by event, although science and system level autonomous capabilities (onboard task planning and scheduling, autonomous navigation, hardware health assessment) have been demonstrated on terrestrial prototypes and Earth-orbiting spacecraft, e.g. EO1[Chien 2005] and ASTERIA[Fesq 2019]. Various stages of planetary missions (e.g. descent and landing, science sequencing etc) have been evolving toward this goal, but the overall system level autonomy - the autonomous management of the vehicles and operations such as sub-surface drilling - has been lacking. The future of OW exploration will demand autonomous operations both for Ice Giant missions and particularly for exploring the deeper sub-surface.

[Reeves 2020] describes the necessary features of an autonomous system for Europa Lander to increase the operational efficiency of a mission, helping to conserve engineering resources while simultaneously increasing the science return of a mission. The overall system that achieves these characteristics must be designed from conception to support autonomy. In the next decade, these autonomous operations will need to be expanded to include ever more complex maneuvers [Amini 2020]. NASA should continue to fund autonomy research together with ground and flight testbeds (particularly for system level autonomy) to enable OWs missions of the future.

\subsection{High Performance, Low Power, Radiation-Hardened Computing and FPGAs}

NASA's High-Performance Spaceflight Computing (HPSC) project is developing flightcomputing technologies that will provide at least 100 times the computational capacity compared to current spaceflight computers. However, as of late summer the project is being revamped, so the need for such a flight technology remains and this should be underscored by the Decadal Survey since high performance computing is required for a broad range of future missions. One of the key requirements of this system for OWs, however, is to be able to operate at reduced power, so that when it is not as readily available, the system can down-regulate. Additionally, a pressing need for future systems is radiation-hardened, high capacity, high-performance memory to support the HPSC, and to support communications, instruments, and other subsystems throughout the 
spacecraft. Also required are a radiation-hardened, high- capacity, high-performance FPGA's and ASICS to implement ancillary processing in instruments and throughout the spacecraft.

\subsection{Landing and Lander Technologies}

Advances in a variety of spacecraft and instrument technologies are required to enable many of the investigations outlined for OWs. In addition, there are many instrument-specific advances that will be needed. The following addresses the general needs for access to specific areas on the surfaces of OWs, operation in cold temperatures with limited power budgets, sample acquisition and preservation, some of which also applies to instruments. Instrument sample handling and mechanisms share needs with other spacecraft mechanisms to operate at the ambient temperatures of OWs, as well as to operate with a high degree of autonomy due to the limitations on communication with Earth. Key technology needs have been recognized in the following areas and are discussed in detail in the many cited white papers e.g. [Schmidt 2020].

\subsubsection{Precision Landing and Landing Hazard Avoidance}

These capabilities have been under development for over a decade for missions to Mars and the Moon and now for Europa Lander and Terrain Relative Navigation (TRN) will be operational on Perseverance. Onboard landing hazard detection capabilities have also been under development, using LIDAR sensors to create high resolution topographic maps of the landing site onboard the descent vehicle in real-time. Precision landing and landing hazard detection systems are very computationally intensive, so FPGA-based coprocessors have been developed for them. This same approach to precision landing is applicable to airless OWs with modest evolutionary changes, because airless bodies allow similar orbital reconnaissance at relatively high resolution. The surfaces of some OWs, particularly Europa, are thought to be rough enough that onboard landing hazard detection during descent is essential. The Europa lander pre-project has already begun to address these needs [Crum 2020], which lays the groundwork for other airless bodies.

Titan is a unique case, because its thick, high, hazy atmosphere limits orbital imaging of the surface to spatial resolutions that are orders of magnitude lower than is possible for Mars and airless bodies and Dragonfly will have to address precise guidance to one of many potential touchdown targets (initially inter-dunes) that are widely distributed in a larger overall landing ellipse ("multi-target" scenario). The potential need for higher fidelity landing hazard detection and avoidance is very dependent on the nature of the landing site. Some sites, such as lakes, seas, or plains, may not need this capability; others such as dry lakebeds, may, so future missions to Titan would need to achieve more precise landing.

\subsubsection{Subsurface Ice Acquisition and Handling}

Depth regimes can be divided into several distinct zones: near-surface (a few $10 \mathrm{~s}$ of $\mathrm{cm}$ ), a few meters (e.g. $<2 \mathrm{~m}$ ), $10 \mathrm{~m}, 10 \mathrm{~m}$ to $1 \mathrm{~km}$, and 1 to $20 \mathrm{~km}$. Examples of near-surface excavation systems include the drill on the Mars Science Laboratory (MSL) mission, Luna 16/20 drills, and Venera/Vega drills. Other approaches such as scoops, harpoons, saws, piercing blades, etc. also fall into this regime and Europa Lander is using bi-blades to remove the irradiated top $\leq 20 \mathrm{~cm}$ from the surface before sampling the pristine material below [Crum 2020]. Many of the white papers submitted to this Decadal Survey summarize the methodologies available to sample deeper into the sub-surface of OWs (see e.g., [Schmidt and Craft 2020], [Carpenter 2020], [Carpenter 2020]). Of note is the need for a variety of approaches for the specific science investigations on each of the OWs. Many of these approaches require that concomitant technology developments be carried out, where the form factor of the surface mobility or sub-surface system dictates the design of instruments and sub-systems contained within it. Three technologies all must have, are 
enough power to operate, a viable communication scheme, often with a back-up e.g. see [Schmidt and Craft 2020] and the ability to act autonomously for sometimes long periods of time.

\subsubsection{Low Temperature Actuators and Mechanisms}

Mechanisms capable of reliably operating at extremely low temperatures are required to enable efficient sample acquisition on Europa. In the absence of improved technologies and/or approaches, thermal control of the acquisition mechanisms will require the use of heaters and advanced thermal insulation approaches. These mass penalties can potentially be eliminated, or the impact reduced, through the development of low temperature mechanisms e.g., bulk metallic glasses. This could be enabling for missions to OWs, such as Europa and Enceladus, where power is a precious commodity and there are significant contamination control and/or planetary protection concerns. A more complete description of the technical goals for actuators and mechanisms are given in the ROW report.

\subsubsection{Instrument Technologies for Ocean Worlds}

The Roadmap presents an ambitious and compelling vision for the study and characterization of OWs, including the searches for bio-signatures. These science goals will be accomplished only with spacecraft carrying a "suite of relevant instruments," with a range of measurements including those for bio-detection, chemical analysis, crystallography, spectroscopy (remote sensing and/or in situ), and gravity field mapping identified as relevant for the various bodies. There are many types of instruments that can fulfill the measurement goals of OWs, but all benefit from having low mass, power and volume. Many of these instruments could benefit from modern use of machine learning [Azari 2020] and science autonomy. Even though the number of planetary science publications involving machine learning has grown exponentially over the last ten years, it lags in both percent share and growth rate compared to other SMD divisions. Science autonomy is the capability of a robotic system to choose for itself which scientific data to collect or, having collected it, which data to send to Earth. Autonomous Exploration for Gathering Increased Science (AEGIS), which is intelligent targeting software, has been in use on MSL since 2016 [Francis 2018] and is a pathfinder for future missions.

\subsection{Ice Sample Return and Backward Planetary Protection}

Returning ice samples from an OW, such as from the plumes of Enceladus, requires a carefully thought out systems approach ensuring the sample acquisition methodology functions flawlessly with an Integrated Cryogenic Chamber (ICC) and a Backward Planetary Protection (BPP) system - all designed to operate together. BPP technologies and procedures are currently under development for the planned Mars Sample Return (MSR) mission campaign, but without maintaining cryogenic temperatures. No mission has returned actively cooled samples. The Stardust and Genesis missions returned passively cooled samples to Earth, but allowed samples to reach $50^{\circ} \mathrm{C}(323 \mathrm{~K})$ during atmospheric entry. Both ICC and BPP technologies are required to preserve samples for $>6$ years for transportation from an OW, such as Enceladus, back to Earth. Alternatively, depending on the scientific investigations, samples can be returned at modestly cool temperature, and the volatiles examined on the return flight, as is envisaged in the Ceres Sample Return mission concept [Castillo-Rogez, 2020].

\section{Technology Recommendations}

Since the Europa Lander pre-project is developing many of the needed technologies for OWs, this should continue to be funded. In addition, the overarching need for many of the required technologies for OWs is the ability to test the components/subsystems in a relevant environment. Field testing of subsystems in representative environments is critical. Many of the OW surface conditions are poorly understood, so those field tests serve to verify operational and autonomy 
protocols, but no field test on Earth is truly going to be representative of conditions. This means that representative testbeds must also be built and maintained to meet those needs. Similarly,

For Ocean World missions, NASA should continue investing in Europa Lander mission concept technologies and fund key developments both for a Neptune (Triton) flagship mission and for innovative new concepts to land and sample on and below the surface of Ocean Worlds.

autonomy testbeds on the ground and in space need to be developed to facilitate development of new algorithms both for system level and for functional level autonomy.

3. References

3.1 2020 Decadal Survey White Papers

Schmidt and Craft 2020, Subsurface Needs for OWs; Carpenter 2020, Venture Deep, the Path of Least Resistance: Crevasse-Based Ocean Access Without the Need to Dig or Drill; Carpenter 2020, The Science Case for Surface Mobility on Icy Worlds ; Crum 2020, Advanced Technology Developments for Europa Lander and other In-Situ OW Missions; Reeves 2020, Mission Autonomy for the Europa Lander Mission; McEnerney 2020, Enabling New Science Mission Capabilities with Additive Manufacturing Technologies; Austin 2020, Enabling and Enhancing Science Exploration Across the Solar System: Aerocapture Technology for SmallSat to Flagship Missions; Benardini 2020, Molecular Biology Modernizing Planetary Protection Engineering to Enable Science for Biologically Sensitive Robotic and Human Missions; Woerner, 2020 (including co-authorship by the DOE Idaho National Laboratory). Radioisotope Heat Sources and Power Systems Enabling OWs Subsurface and Ocean Access Missions; Austin 2020 Enabling and Enhancing Science Exploration Across the Solar System: Aerocapture Technology for SmallSat to Flagship Missions; R. Bugga 2020, Energy Storage Technologies for Planetary Science and Astrobiology Missions; A. Azari 2020, Integrating Machine Learning for Planetary Science: Perspectives for the Next Decade. R. Amini 2020, Advancing the Scientific Frontier with Increasingly Autonomous Systems.

3.2 Other References

P. Stella et al., 2009,"LILT testing of solar cells for the Juno (Jupiter orbiter) mission", $21^{\text {st }}$ Space Photovoltaic Research and Technology Conference; S. Dawson et al., 2012,"Juno photovoltaic power at Jupiter", $10^{\text {th }}$ International Energy Conversion Engineering Conference; M. Kroon et al, 2019, "Solar arrays for Jupiter missions Europa Clipper and JUICE", $37^{\text {th }}$ Space Power Workshop; P. Chiu et al, 2019, "Qualification of 32\% BOL and 28\% EOL efficient XTE solar cells", 46 ${ }^{\text {th }}$ IEEE Photovoltaics Specialists Conference; C. McPheeters et al, 2018, "High efficiency multijunction solar cells for LILT applications, Conference on Advanced Power Systems for Deep Space Exploration; A. Boca et al, 2017, "A data-driven evaluation of the viability of solar arrays at Saturn", Journal of Photovoltaics vol. 7, issue 4, pp. 1159-1164; A. Boca et al, 2019, "Solar power for deep-space applications: state of art and development", AIAA Propulsion-Energy Forum; T. R. Spilker et al, 2019, An Assessment of Aerocapture and Applications to Future Missions, Journal of Spacecraft and Rockets Vol 56 No 2 pp 236-24 doi:http://arc.aiaa.org/doi/abs/10.2514/1.A34056; R. Francis et al, 2017, AEGIS autonomous targeting for ChemCam on Mars Science Laboratory; deployment and results of initial science team use., Science Robotics v2,(7). Chien $\mathbf{S}$ et al, 2005, Using autonomy flight software to improve science return on Earth Observing One. Journal of Aerospace Computing, Information, and Communication. Apr;2(4):196-216. Fesq, L., et al., 2019 "Extended Mission Technology Demonstrations Using the ASTERIA Spacecraft," IEEE Aerospace Conference, Big Sky, MT, March doi: 10.1109/AERO.2019.8742020, https://ieeexplore.iee.org/document/8742020/. 